Arqueología y Sociedad,

№ 22, 2010

\title{
RELECTURA DE FUENTES PRIMARIAS PARA LA DOCUMENTACIÓN DE GEOGLIFOS EN EL VALLE BAJO del Río Chillón, Costa Central del Perú
}

Jorge Carranza Orbegoso*

\section{Resumen}

Recientes investigaciones han evidenciado la presencia de geoglifos en pampas y quebradas que rodean las cuencas inferiores de los valles de la costa central del Perú. El propósito de este documento es evaluar la información reunida hasta el momento y añadir nuevos datos sobre tres sitios arqueológicos con presencia de geoglifos, ubicados en la margen izquierda del valle bajo del río Chillón, inexistentes en la actualidad, destruidos por el avance urbano de la ciudad capital, localizados mediante la revisión de antiguas fotografías aéreas y la cartografía histórica existente del área. Se describen los geoglifos en relación a sitios arqueológicos y rutas que conectan el valle bajo del río Chillón y Pampa Canto Grande, lugar donde se ha ubicado uno de los mayores centros de Arte Rupestre de la zona.

Palabras clave

Perú, Costa central, valle del río Chillón, Arte rupestre, geoglifos, Collique, Comas, Canto Grande.

\begin{abstract}
Recent investigations show the presence of geoglyphs in pampas and canyons around the down basins of the central coast valleys of Peru. The purpose of the present work is to evaluate the collected information and to give some additional details about three archaeological places in the left margin of the valley of Chillon river. These places don't exist any more, because the urban development, and they were found thanks to old aerial photos and historical cartographie of the zone. The geoglyphs are described in relation to the archaeological places and routes connecting the down valley of the Chillon river and the Canto Grande Pampa, where one of the biggest rock art centers can be found.
\end{abstract}

Keywords

Peru, Central coast, Chillon river valley, Rock art, geoglyphs, Collique, Comas, Canto Grande.

\footnotetext{
* Bachiller en Arqueología. Universidad Nacional Federico Villareal.

Correo electrónico: antoinecarranza@yahoo.com
} 
... Estos templos y adoratorios ... estaban unos en poblado y otros por los campos, sierras y montañas agrias; unos en los caminos, y otros apartados dellos; en los sembrados y tierras de labor, y en punas y desiertos y dondequiera ... No todos los adoratorios eran templos y casas de morada; porque los que eran cerros, quebradas, peñas, fuentes, y otras cosas a este tono, no tenian casa ni edificio, sino cuando mucho un buhio o choza, en que moraban los ministros y guardas de las dichas guacas ...

Bernabé Cobo (1956:167-168[1653])

\section{INTRODUCCIÓN}

El presente artículo, detalla una serie de referencias sobre la presencia de geoglifos en las quebradas aledañas al valle bajo del río Chillón, en la margen izquierda, donde actualmente se constituye parte del extremo norte de Lima Metropolitana.

La presencia de este tipo de manifestaciones en el valle de Lima (Costa Central), ha sido precisada por diversos investigadores en los últimos años.

Geoglifos, incluidos como una modalidad de arte rupestre, en lo que concierne al valle del río Chillón y sus alrededores, han sido localizados en las inmediaciones del valle medio y bajo, principalmente en los conos deyectivos que forma la cadena de cerros que separan el valle con la quebrada que compone Pampa Canto Grande, perpendicular y afluyente del valle bajo del río Rímac, donde se ha registrado uno de los mayores complejos de geoglifos del área.

Los sitios arqueológicos en cuestión, al presente, se encuentran desaparecidos, destruidos durante los años sesenta por areneras, fábricas y finalmente por asentamientos humanos y urbanizaciones populares, conformando actualmente parte del distrito de Comas.

\section{UBICACIÓN Y ENTORNO GEOGRÁFICO}

El área de investigación se encuentra dentro de la poligonal conformada por las coordenadas UTM(PSAD56): $8684000 \mathrm{~N}, \quad 278000 \mathrm{E}$;
$8684000 \mathrm{~N}, 282000 \mathrm{E} ; \quad 8676000 \mathrm{~N}, 276000 \mathrm{E}$; $8676000 \mathrm{~N}, 280000 \mathrm{E}$; constituida principalmente por tres quebradas contiguas, que descienden en dirección este-oeste, situándose en el lado occidental de la cadena de cerros que separa el piso del valle con Pampa Canto Grande, en la margen izquierda del valle bajo del río Chillón.

Los puntos altitudinales fluctúan entre los 100 y 450 msnm, perteneciendo cada nivel al límite con el valle y el extremo oriental de las pampas, en el interior de los cerros, respectivamente, ubicándose en la región ecológica denominada costa desértica, valle bajo o yunga (0-600 msnm). Esta región presenta una neblina baja que permite la formación de lomas, localizándose actualmente relicto de éstas en los cerros del valle bajo del río Chillón, incluidos dentro del radio urbano de Lima.

Al presente, el área de estudio se ubica al lado este de la avenida Tupac Amaru (antiguamente llamada carretera Lima-Canta) entre los kilómetros 11 y 18 aproximadamente, siendo ocupada por asentamientos humanos y urbanizaciones populares, formando parte del distrito de Comas, en el sector conocido como Lima Norte, en la provincia y departamento de Lima ${ }^{11}$.

1 La ubicación de los sitios arqueológicos se realizó mediante el auxilio de la cartografía histórica existente para el área (Plano referente al valle de Lima, escala 1:300,000; fechado en 1925, al parecer de carácter inédito, elaborado por Camilo Vallejos Z. para la Sociedad Geográfica de Lima [SGL]; Archivo Francisco G. Vallejos Berríos [AFGVB]; Carta "Lima y alrededores", escala 1:50,000; impresa en 1937, del Servicio Geográfico del Ejercito [SGE]; Mapoteca del Centro de Investigaciones Histórico-Militares del Perú [M-CIHMP], y la "Carta de Lima y alrededores [Hoja 5f 'Infantas', Hoja 6f 'Comas']”, escala 1:20,000; actualizada y reimpresa en 1962 del Instituto Geográfico Militar [IGM]; Archivos de la Dirección General de Geografía del Instituto Geográfico Nacional [ADGG-IGN]), levantadas por procedimientos y métodos convencionales, las cuales proporcionan importantes datos toponímicos y arqueológicos, producto del contacto directo de los operadores con el terreno y sus pobladores, todo esto para complementar la información de la cartografía actual del IGN. El trabajo incluye la revisión de fotografías aéreas oblicuas y verticales (Servicio Aerofotográfico Nacional [SAN] O-764 [elevación 1850 pies, 26 de Abril de 1943], O-4225 [elevación 1000 pies, 5 de Octubre de 1944], y el Proyecto de vuelo 1962-1963, foto $\mathrm{N}^{\circ} 243$ [escala 1:20,000; 1 de Abril de 1962] del IGN). 


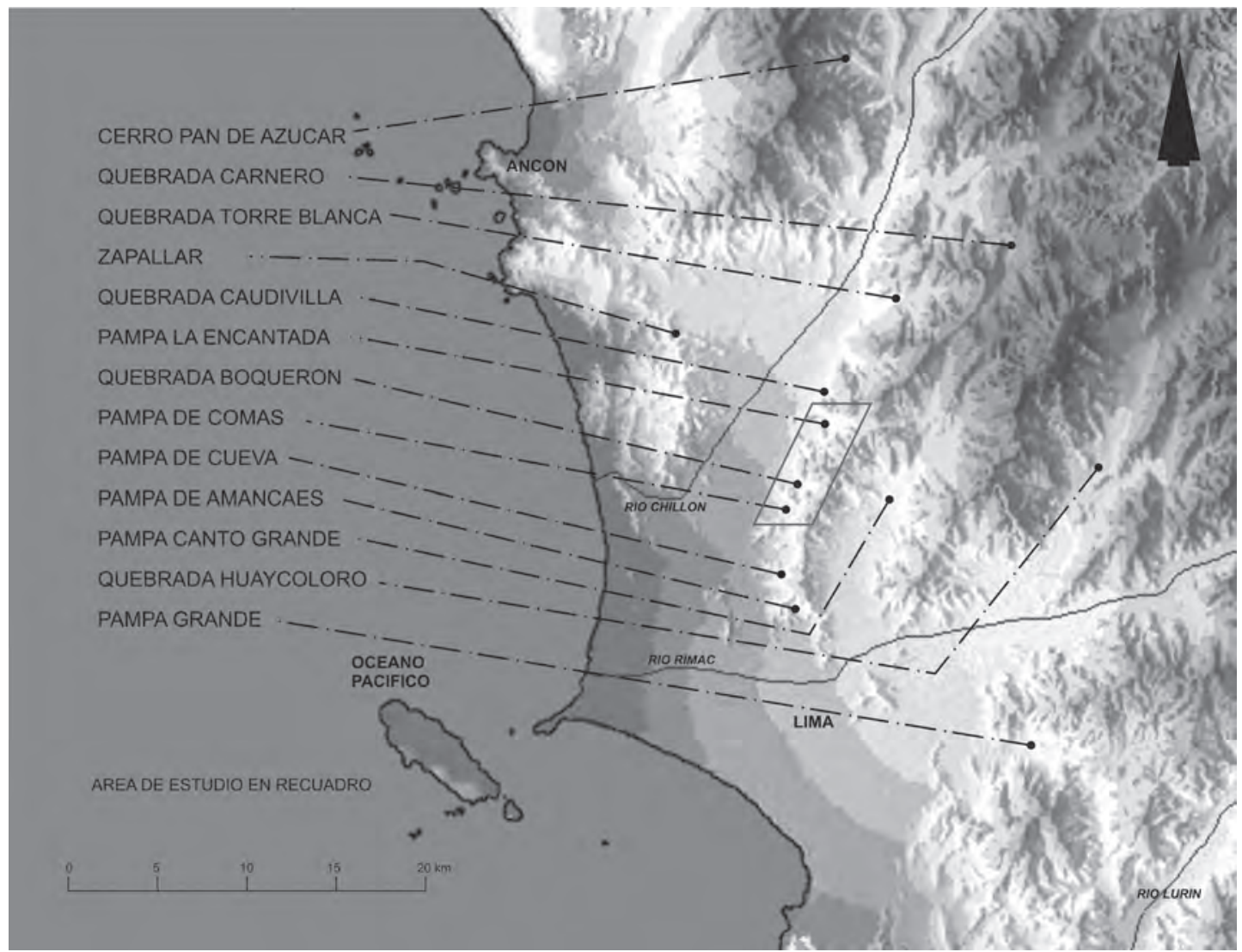

Figura 1. Geoglifos de los valles bajos de los ríos Rímac y Chillón, costa central del Perú. Mapa adaptado de Falling Rain Genomics, Inc (1996-2004).

\section{ANTECEDENTES DE INVESTIGACIÓN}

La situación de Pampa Canto Grande, entre las cuencas inferiores de los ríos Rímac y Chillón, propició la utilización de quebradas y pampas vecinas, como es el caso de las ubicadas en el lado occidental de la cadena de cerros que separa el valle bajo del río Chillón con la Pampa Canto Grande (Fig. 1).

Las investigaciones en el área de estudio concerniente a geoglifos son escasas y, en su mayoría, breves menciones y sucintas descripciones. A continuación, aludimos datos de trabajos realizados por diversos investigadores que involucran el área de estudio y sectores contiguos, dentro de la zona de influencia.

Horkheimer (1947: 54), sobre la base de una comunicación del Capitán Fernández del Escua- drón 23 de la Aviación Militar Peruana, menciona la presencia de líneas en el área de Canto Grande, ubicando en el archivo del SAN algunas fotos (O-2518 y O-2522) las cuales mostrarían los geoglifos en Pampa Canto Grande².

Por otro lado, Reiche (1993: 178[1949]) indica haber recibido información de las líneas en Pampa Canto Grande, por parte del ingeniero Kanmacher, quien las conociera desde finales de la década de 1920. También señala la presencia de trazos, en la quebrada que se ubica al este

2 Roselló (1997: xvii, Lámina I, Lámina II) presenta dos fotografías aéreas del SAN bajo los códigos O-2518 y O-2526 que refieren a Pampa Canto Grande. La fotografía aérea con el código O-2522, citada por Horkheimer pertenece al Cerro Pan de Azúcar, ubicado en la margen derecha del valle bajo del río Chillón (Rodríguez 1997:50, Figura 5). 
de la Escuela de Aviación Civil Collique y en la quebrada inmediata al sur de ésta, la que compone Pampa de Comas.

En el año 1960, Horkheimer en compañía del Dr. Georg Petersen Gaulke y el geógrafo Gonzalo De Reparáz Ruiz, realiza un reconocimiento de la parte baja de Pampa Canto Grande (Roselló 1997: xi). También Kosok (1965: 57) precisa haber sido llevado a Pampa Canto Grande por Thommen, en compañía de Reiche ${ }^{3}$.

Años más tarde, Horkheimer (1965: 51) en la primera parte de un inventario de sitios arqueológicos, informa sobre la presencia de plazoletas en la quebrada que se encuentra al sur de Cerro Fortaleza, aludiendo específicamente a Pampa La Encantada. De igual forma comunica la presencia de estructuras similares en quebradas contiguas.

En 1970 Mazzotti, ex jefe de Astronomía del IGN, publica un artículo periodístico mostrando un esquema de algunas de las rayas de la parte baja de Pampa Canto Grande, en el diario "El Comercio" de Lima (Roselló et al. 1985: 43; Roselló 1997: xi).

Provistos de este material, el Seminario de Arqueología del Instituto Riva Agüero, de la Pontificia Universidad Católica del Perú, a cargo de la Dra. Josefina Ramos de Cox, exploran y señalan la presencia de geoglifos en Quebrada Torre Blanca y Pampa Canto Grande, derivando un informe entregado al entonces Patronato Nacional de Arqueología, por la Dra. Josefina Ramos de Cox, fechado el 2 de Noviembre de 1971 (Morrison 1978: 82-85; Roselló 1997: xi, 37-38; Roselló et al. 1985: 41; Rostworowski 1972: 323-324) ${ }^{4}$.

A partir de la segunda mitad de la década de 1950, Roselló realiza una serie de reconocimientos arqueológicos de los valles bajos de

3 Paúl L. Thommen, representante de Fairchild Aerial Surveys en el Perú, había mostrado interés en el tema años antes (Reiche 1993; Thommen 1946).

4 Pampa Canto Grande recibe mayor atención por parte de diversos investigadores a finales de los años sesenta en adelante. (Bonavia 2000; Hostnig 2003; Mazzotti 1973; Milla 1974; Pelikan 1968; Ravines 1978). los ríos Rímac y Chillón, contando con la colaboración de diversos investigadores del medio (Roselló 1978, 1997; Roselló et al. 1985)5 . Por esos años Roselló, acompañado de Horkheimer, afirma el avistamiento de geoglifos en la pampa que compone la quebrada que se ubica al este de Cerro Fortaleza (Roselló 1997: xiii, 37, 76). Conjuntamente con Mejía, en 1956, exploran la gran raya de Pampa de Comas (Roselló 1978: 522; 1997: xi, 10, 42-43). En el año 1971, registra los geoglifos de Quebrada Torre Blanca (Roselló 1978:523; 1997:37-38). En 1976 Roselló, Huapaya y Mazzotti inician el "Proyecto Canto Grande", viéndose interrumpido en 1980 (Caretas 1995; Roselló 1997; Roselló et al. 1985; Zileri 1995). Es importante indicar que Roselló, mediante el análisis de fotografías aéreas, señala a Quebrada Caudivilla y Pampa de Amancaes, como sitios arqueológicos con presencia de geoglifos (Roselló 1997: xii-xiii).

En 1962 Mejía, quien décadas antes diera a conocer sus estudios de los geoglifos de la región del Río Grande de Nazca (Mejía 1942), en compañía de Jorge C. Muelle Rojas, Vicente Segura Núñez y José Casafranca Noriega, realiza una inspección al sitio arqueológico Pampa de Cueva, advirtiendo la presencia de una raya en composición del sitio arqueológico (Mejía 1978: 508). Engel (1983) manifiesta haber observado geoglifos en el área donde se sitúa la urbanización popular Comas, durante los años cincuenta ${ }^{6}$.

Algunos años más tarde, las investigaciones de Rodríguez (1997) señalan para el valle bajo del río Chillón como sitios arqueológicos con presencia de geoglifos a Quebrada Torre Blanca, Zapallar, Cerro Pan de Azúcar, Collique, Quebrada Repartición, y Pampa de Cueva. Posteriormente, presenta los resultados de un reconocimiento en Quebrada Torre Blanca, constatándose la existencia de estructuras estre-

5 Ver también El Comercio (1977); López (1983); La Prensa (1980); Silva (2002); Valls (1980).

6 Petersen (1980:26) menciona como sitio arqueológico con presencia de geoglifos a Quebrada Repartición; Pampa Repartición y la quebrada que conforma se ubica contigua al lado sur de Pampa de Comas. La descripción que realiza del sitio arqueológico, evidencia la confusión de nombres con Pampa de Comas. 
chamente relacionadas con Pampa Canto Grande (Rodríguez 1999)?

Después de una serie de exploraciones arqueológicas realizadas en la quebrada que conforma Pampa Canto Grande y sus afluentes, Abanto desarrolla el proyecto "Prospección Arqueológica en la Parte Alta de la Quebrada Canto Grande", registrando noventa sitios arqueológicos, cuarenta de los cuales corresponden a geoglifos. Abanto informa de un complejo de geoglifos en Quebrada Carnero, colindante en su vértice con Quebrada Canto Grande y afluente al valle bajo del río Chillón (Abanto 2003, 2004; Abanto y García-Godos 2004).

Bajo un proyecto de inventario de sitios arqueológicos, Tosso (2003) identifica una confluencia de estructuras hacia el este de Pampa de Comas, en las inmediaciones de Cerro Almoguera denominándolo Complejo Interdistrital, el cual describe como escalinatas, terrazas, muros, corrales y alineamiento de piedras a manera de recintos; elementos que recuerdan lo registrado en Pampa Canto Grande y sus afluentes (Abanto 2003, 2004; Abanto y García-Godos 2004).

Por otro lado, Roselló al referirse a la margen izquierda del valle bajo del río Chillón, advierte la presencia de ligeros promontorios, conocidos mayormente como conglomerado de montículos, los cuales aparecen relacionados a los geoglifos y se ubican en el piso del valle (Roselló 1997: 10).

Para el valle medio del río Chillón, se han señalado como sitios arqueológicos con presencia de geoglifos a Cerro Pinchausa (CIPMFAUAUNI 1988:II:Ficha 0064; Horkheimer 1965:50),

7 Ver también Centro de Investigación del Patrimonio Monumental. Facultad de Arquitectura, Urbanismo y Artes-Universidad Nacional de Ingeniería [CIPMFAUA-UNI] (1988:III:Ficha 0168, 0218, 0297); Fondo Documentario de la Cultura Peruana-Universidad Nacional Federico Villarreal [FDCP-UNFV] (1998); Morrison (1978: 82-85); Necochea (1994); Rostworowski (1972: 323-324). Para el caso de la confluencia de los sitios arqueológicos Zapallar-Copacabana, se ha documentado un geoglifo en las cercanías (CIPMFAUA-UNI 1988: III: Ficha 0179).

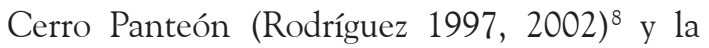
Quebrada Quilca, en las inmediaciones de la casa hacienda homónima, antigua ruta que conduce a Huaral, valle inferior del río Chancay (Horkheimer 1965: 48) ${ }^{9}$.

\section{Geoglifos de Pampa La Encantada}

\section{Ubicación}

Sitio arqueológico actualmente desaparecido, noticias sobre el mismo, llegan a nosotros a través de las investigaciones de Horkheimer (1965: 51) y Roselló (1997: xiii, 37, 76), que señalan a la quebrada vecina al sureste de Cerro Fortaleza, a la altura del kilómetro 16 de la avenida Túpac Amaru, aludiendo específicamente a Pampa La Encantada.

Pampa La Encantada es la llanura aluvial que conforma este cono deyectivo, delimitada naturalmente por el oeste con el piso del valle, por

8 Véase también los trabajos de CIPMFAUA-UNI (1988: II: Ficha 0067); Necochea (1993) y Silva (1996: 257). Brignardello (2000: 231-232, 2004: 125, 132-135, 144-145, 2006: 37-47) documenta un centro de arte rupestre en Quebrada Tinajas, valle bajo del río Lurín, lugar donde se a identificado un geoglifo, con la misma temática al registrado en Cerro Panteón. Cabe mencionar que Brignardello exploró desde el año 1997 la Quebrada Huaycoloro (Valle bajo del río Rímac), Quebrada Tinajas, Quebrada Buenos Aires y Quebrada Chamallanca (Valle bajo y medio del río Lurín), señalando la presencia de geoglifos en las mismas.

9 En relación a este último sitio arqueológico con presencia de geoglifos, queremos centrar la atención en las investigaciones toponomásticas realizadas por Pulgar, Director del Departamento de Geografía y principal del Departamento de Investigaciones Toponímicas de la Facultad de Letras de la Universidad Nacional Mayor de San Marcos, iniciadas a mediados de la década del cuarenta en el área andina. Señalan al vocablo quechua quilca y sus variantes fonéticas, relacionadas al Arte Rupestre (Pulgar 1962, 1976). Las voces quilca o quellca subsisten solas o asociadas a otros vocablos, por medio de la cual los pobladores andinos se referían a una marca gráfica o una forma o medio de expresión. Posterior a la llegada de los españoles, quilca fue interpretada en los diccionarios coloniales quechuas como escribir, dictar, registrar, pintar, dibujar, labrar, esculpir, rasgar, bordar; por defecto a personas y objetos relacionados a estas actividades. 
su lado norte con Cerro Fortaleza, Cerro Gentiles, Cerro Vaca, Cerro El Zorro y la Loma de San Antonio, por el lado sur con Pampa Captación Norte, Cerro Las Minas, Quebrada Zorro Blanco, Cerro Ladrón, los Cerros de Collique y Cerro Punta Aguja en las Alturas de Zorro Blanco.

El interior de Pampa La Encantada se subdivide en: Quebrada de Los Lomeros y Quebrada de Collique ${ }^{10}$. Al presente, se ubica en Pampa La Encantada y la quebrada que conforma, la urbanización popular Collique ${ }^{11}$.

\section{Descripción}

La quebrada que conforma Pampa La Encantada posee una extensión aproximada de cuatro kilómetros de longitud y un ancho variable de medio kilómetro. Topográficamente, el área presenta un relieve plano, inclinado al oeste y ligeramente ondulado. Posee una altitud que va entre los 170 y $430 \mathrm{msnm}$.

Los primeros reportes sobre geoglifos en Pampa La Encantada nos lo brinda Horkheimer, señalando que: "En la quebrada al S [Sur] del peñón [Cerro Fortaleza] se observan plazoletas similares a las que forman parte en el complejo de las marcas de Nazca. Otras hubo en quebradas vecinas, pero fueron destruidas por urbanizaciones y otras ocupaciones modernas" (Horkheimer 1965: 51).

Es importante indicar que Roselló (1997:37) afirma este avistamiento, indicando que estas rayas le fueron mostradas por Horkheimer durante los años cincuenta ${ }^{12}$.

10 Fuente: Carta "Lima y alrededores" 1:50,000; 1937, SGE (M-CIHMP); "Carta de Lima y alrededores (Hoja $6 f$ 'Comas')" 1:20,000; 1962, IGM (ADGG-IGN).

11 Pampa La Encantada se encuentra dentro del área conocida como Collique. El topónimo Collique hace referencia a un asentamiento prehispánico tardío en la margen izquierda del valle bajo del río Chillón, entre los kilómetros 16 y 18 de la avenida Tupac Amaru, cubriendo un área de 20 a 25 hectáreas hacia el piso del valle. El componente principal del sitio arqueológico seria la denominada "Fortaleza de Collique" del cual deriva el nombre del cerro donde se emplaza.

12 Ludeña (1975:48), dejando entre ver su existencia, sugiere que estos geoglifos pudieron encontrarse en
Según la definición de plazoleta que precisa Horkheimer en un trabajo referido a los geoglifos de la región Palpa-Nasca (Horkheimer 1947), en rasgos generales, señala áreas de forma triangular, rectangular y trapezoidal, trazadas en terreno plano o de declive suave, elaboradas mediante la limpieza del área, acumulando las piedras en los bordes a manera de resaltar la línea y formando pequeños montículos. También, en uno de sus extremos existiría una construcción baja.

Se tiene un grupo importante de datos, identificados a inicios de la década de 1970, referidos al extremo suroeste de Pampa La Encantada, en la llanura próxima a la ladera oeste del Cerro Las Minas y Pampa Captación Norte, donde a la fecha, ya se encontraría instalado parte del Asentamiento Humano Año Nuevo. Los autores coinciden en señalar la presencia de estructuras y material asociado atribuible al precerámico (Huapaya 1977-1978: 30; Ludeña 1975: 48, 196, 201; Roselló 1997: xxi, 9, 71; Roselló et al. 1985: 57; Silva 1996: 130-131, Figure III PV46-135; Tosso 2003).

Tosso (2003), registra una serie de estructuras hacia el interior de Pampa La Encantada (Lomas de Collique, Sitio Mirador y Sitio Refugio), en Cerro Zorro, Loma de San Antonio, Quebrada de Los Lomeros y Quebrada de Collique, definidas como estancias y corrales, posiblemente relacionados a las lomas y la actividad que derivaba de esta ${ }^{13}$. Estructuras similares han sido registradas en Punchauca, Quebrada El Progreso (Ludeña 1975: 54-55), Quebrada Torre Blanca (Rodríguez 1999: 309, 311), Cuello de Collique (Abanto 2003: 31), en las cercanías de Cerro Cucaracha, valle bajo del río Chillón y Cerro Cabeza de León en la Pampa Canto Grande (Roselló 1997: xxiii-xxiv). La cronología es

Pampa La Encantada. El sitio arqueológico de Pampa La Encantada recibe especial mención por parte de Hostnig (2003) y Rodríguez (1997) denominándolo Collique.

13 En referencia al área urbanizada (Collique, V Zona) se tiene un proyecto de reconocimiento en la modalidad de evaluación arqueológica (Pinto 2001), pero no se detalla mas de lo registrado anteriormente. 


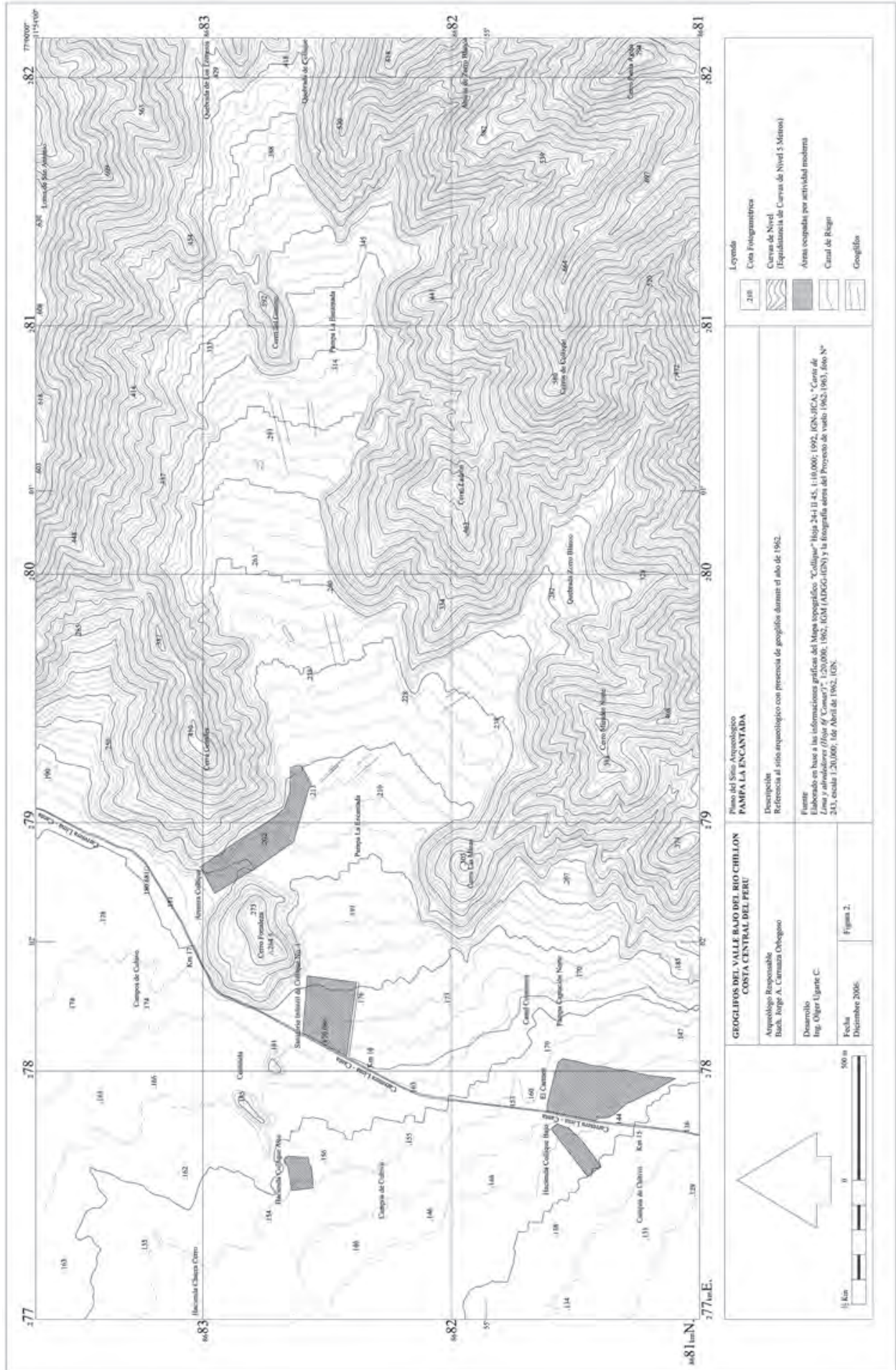

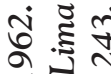
$\approx$

ฉี है है

$\tau$

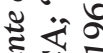

छิ

녕은

t5

ऽू इ

$\approx$ ะ

.

ठ

竞芒

के भे

$8:=$

$\mathrm{Br}^{\circ} \cdot \mathrm{i}$

$\because \frac{1}{\circ}$

ษั

की

हิ

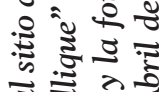

उ

ป 엉

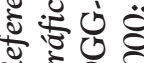

․

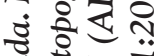

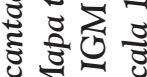

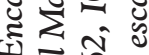

अ

है

है

c.

$\stackrel{8}{*} \stackrel{\sim}{\sim}$

50.5

ठै है

结乱

ปั

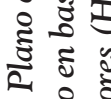

교

है : 
tentativa y va desde tiempos prehispánicos hasta los republicanos (Cogorno 2006, Gutiérrez 1992).

La evidencia observada en las fotografías aéreas del SAN O-4225 (elevación 1000 pies, 5 de Octubre de 1944), e IGN Proyecto de vuelo 1962-1963 foto $\mathrm{N}^{\circ} 243$ (escala 1:20,000, 1 de Abril de 1962) (Figs. 2 y 3), señala un grupo de plazoletas bastante deterioradas que se extienden por Pampa La Encantada, Quebrada Zorro Blanco, hasta las proximidades del Cerro del Camino, en la sección media de la pampa.

La parte compuesta por Pampa La Encantada y Quebrada Zorro Blanco presenta una plazoleta, orientada de noroeste a sureste, elaborada mediante la limpieza del área y definida por dos líneas construidas en base a la alineación y acumulación de piedras, la cual se extiende desde las cercanías de Cerro Fortaleza y Cerro Gentiles, cruzando Pampa La Encantada y ascendiendo por Quebrada Zorro Blanco. Tanto las fotografías aéreas como la cartografía histórica consultada para la ubicación del sitio arqueológico, muestran el área de la plazoleta bastante afectada por las labores de extracción y transporte de una arenera.

La sección de Pampa La Encantada, donde predomina una extensión de Cerro El Zorro denominada Cerro del Camino se ha identificado un conjunto de plazoletas que confluyen en sus cercanías de características similares a la descrita anteriormente. En la margen norte, parte media de la pampa, se definen dos espacios rectangulares asociados a los geoglifos. Es notoria también la presencia de corrales en lugares resguardados, en la base de los cerros.

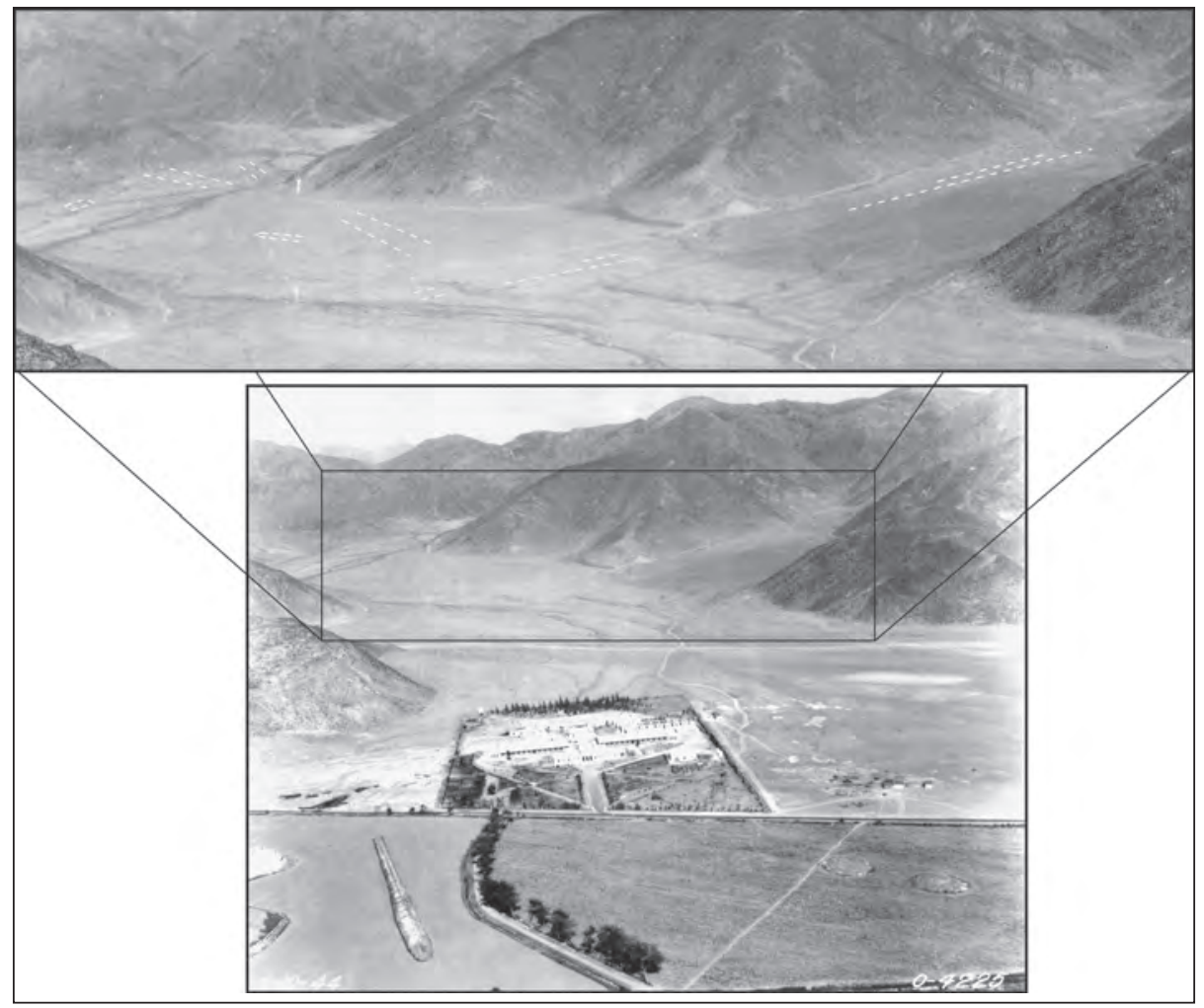

Figura 3. Pampa La Encantada, vista general desde el oeste del sitio. En primer plano el Hospital Sergio Bernales, en ese entonces Sanatorio Infantil de Collique $N^{\circ} 1$, frente a este algunos montículos en el piso del valle. En la sección ampliada se encuentran remarcadas las plazoletas y las estructuras rectangulares que componen este complejo de geoglifos (SAN O-4225 [elevación 1000 pies, 5 de Octubre de 1944]). 


\section{Geoglifos de Pampa de Comas}

Por las referencias encontradas se ha podido determinar dos sitios arqueológicos con presencia de geoglifos, asentados en quebradas contiguas. La denominada propiamente Pampa de Comas y la quebrada vecina al norte, denominada por razones descriptivas Quebrada Boquerón, ubicadas actualmente entre los kilómetros 11 y 13 de la avenida Túpac Amaru ${ }^{14}$.

\section{Quebrada Boquerón}

\section{Ubicación}

Las referencias sobre el sitio arqueológico las obtenemos a través de uno de los primeros folletos de Reiche (1993 [1949]), en el cual ofrece una síntesis del avance de sus investigaciones en las pampas de Nasca y Palpa. Los datos señalan a la quebrada inmediata al norte de Pampa de Comas, conformada en su lado norte con Cerro Cruz General y Cerro Boquerón; por el este con el lado sur de Cerro Mirador, Portillo Malva o Cuello de Comas y Cerro Canto Grande; por el lado sur con los cerros y la pampa de Comas y por el lado oeste con el piso del valle. Actualmente se ubican sobre esta explanada las urbanizaciones populares de La Pascana, El Carmen y Señor de Los Milagros, a la altura del kilómetro 13 de la avenida Túpac Amaru.

\section{Descripción}

Quebrada Boquerón tiene dos kilómetros y medio de longitud y un kilómetro de ancho en su lado próximo al valle. Muestra una pendiente moderada inclinada al oeste, con una altura entre los $150 \mathrm{msnm}$, cerca al piso del valle y 380 msnm, en el interior de los cerros.

14 Las investigaciones históricas demuestran la existencia del topónimo Comas en el área, desde tiempos prehispánicos (Tácunan 2000:290); respecto al nombre de Quebrada Boquerón, este procede del cerro de mayor envergadura que la conforma en su lado norte. Fuente: Carta Lima y alrededores 1:50,000; 1937, SGE (M-CIHMP). Para cuestiones de toponimia relacionadas a la topografía de los sitios arqueológicos de Pampa de Comas y Quebrada Boquerón refiérase a la Nota 10.
En 1949, refiriéndose a la quebrada contigua al lado norte de Pampa de Comas, Reiche anotaba: "En la espalda a ésta [Cadena de cerros que empieza con el Cerro San Cristóbal], al lado del camino a Canta, existen también algunas líneas. Desde el ómnibus o ferrocarril camino a Ancón se pueden ver claramente dos trazos muy anchos, uno que señala hacia el camino a la Escuela de Aviación, otro un poco más al Sur en la quebrada siguiente [Pampa de Comas]" (Reiche 1993: 178[1949]).

Cabe notar de que Reiche, en las descripciones sobre los geoglifos de Nasca y Palpa, asemeja la manufactura de los geoglifos, a trazos que se realizan al dibujar, y cataloga los geoglifos en tres clases de trazos: líneas largas, pistas y figuras. La autora especifica, que las líneas suelen ser rectas y en algunos casos aparecen formando grupos de paralelas o casi paralelas (Reiche 1993[1949]).

En el inventario de sitios arqueológicos auspiciado por la Municipalidad Distrital de Comas, Tosso (2003) ubica un sitio arqueológico en la cumbre del Cerro Cruz General, ocupado actualmente por gran parte del pueblo joven Dios es Amor, del cual deriva su denominación.

Se trataría de una estructura compuesta por dos plataformas superpuestas, de planta rectangular, orientada de noreste a suroeste, patrón similar al registrado en estructuras de Cerro Cantería (Abanto y García-Godos 2004); Cerro Entierro, limite norte de Pampa de Cueva (Silva 1996: Figure 66), Cuello de Amancaes (Roselló 1978: Foto 4; 1997: 73, Lamina III). Tosso (2003) debido al material asociado, asigna la estructura al Horizonte Temprano.

La evidencia observada en la fotografía aérea SAN O-764 (elevación 1850 pies, 26 de Abril de 1943) (Fig. 4) permite afirmar que se trata de una plazoleta, asentada en el lado sur del fondo de la quebrada de pendiente suave, compuesta por dos líneas de aproximadamente un kilómetro y medio de longitud, orientada al noreste y construida mediante la técnica de la extracción descrita para el caso anterior. 


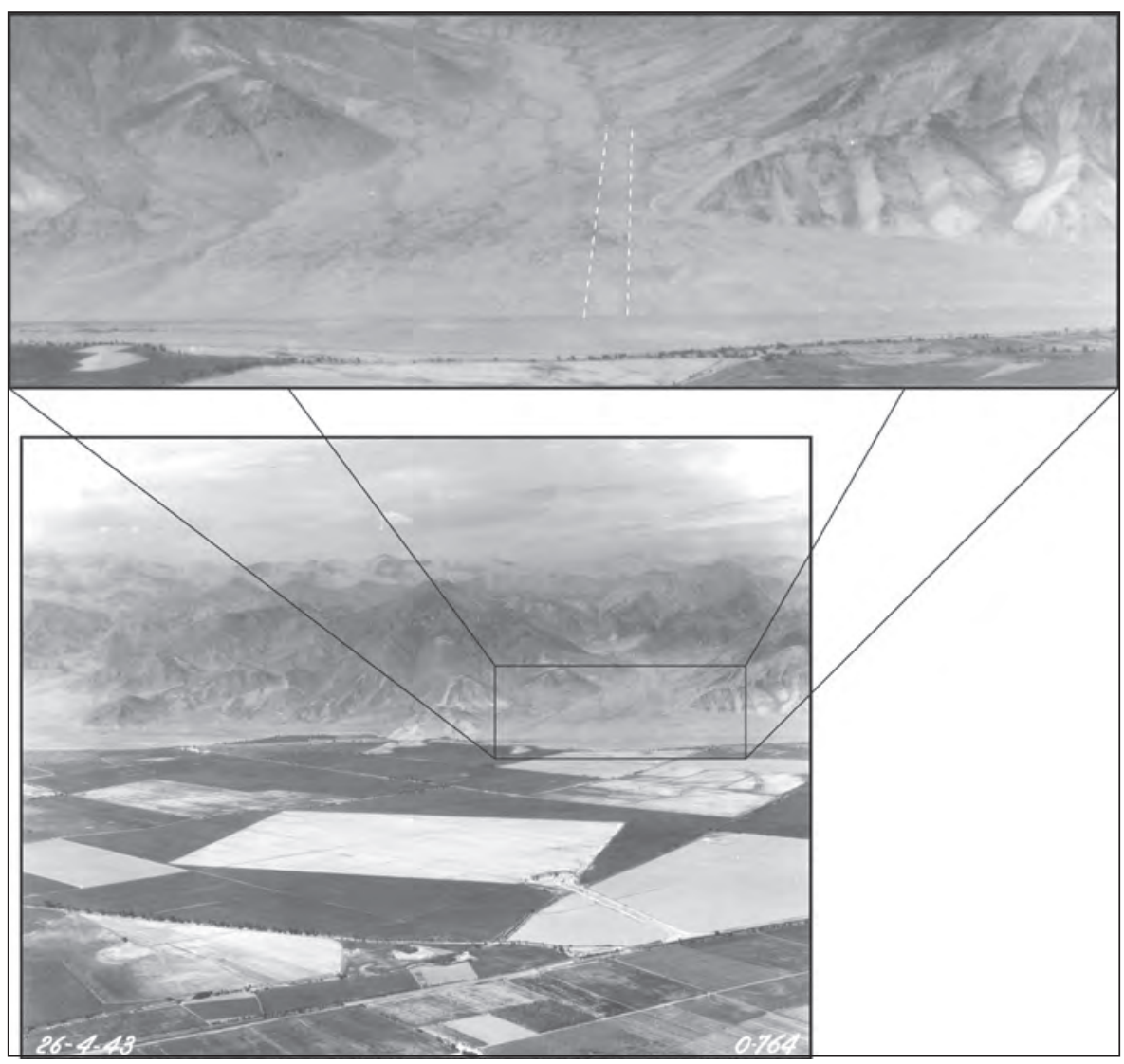

Figura 4. Vista panorámica de Quebrada Boquerón. Antigua fotografía aérea oblicua donde se distingue en el centro inferior de la foto el edificio principal y el área destinada para la Escuela de Aviación Civil Collique. Nótese en el detalle de la fotografía la plazoleta remarcada (SAN O-764 [elevación 1850 pies, 26 de Abril de 1943]).

\section{Pampa de Comas}

\section{Ubicación}

Reconocido por Roselló (1978:5 22; 1997: xi, 10, 42-43) en la década de $1950^{15}$, Pampa de Comas se encuentra limitada naturalmente, en su lado oeste, por el piso del valle, siendo éste su lado más extenso, que se va estrechando conforme va ascendiendo el terreno hasta su extremo este el cual esta limitado por Cerro Canto Grande, Quebrada Piedra Grande y Cerro Almogue-

15 El topónimo Pampa de Comas se encuentra implícito en el plano referente al valle de Lima, 1:300,000; 1925, SGL (AFGVB). Los geoglifos de Pampa de Comas también son mencionados por Engel (1983), Ludeña (1975: 48) y Ravines (1978). Ver también Nota 6. ra. Hacia el lado sur por el Cerro Repartición, al norte por los Cerros de Comas. En la actualidad se ubica sobre esta explanada la urbanización popular La Libertad, a la altura del kilómetro 11 de la avenida Túpac Amaru (Fig. 5).

\section{Descripción}

Pampa de Comas tiene una extensión de dos kilómetros de longitud y un kilómetro de ancho en su lado mayor frente al piso del valle. Presenta un relieve llano, inclinado al oeste, con una altitud entre los 100 y 300 msnm, correspondiendo cada nivel al límite con el valle y el extremo interior de la pampa, respectivamente. 


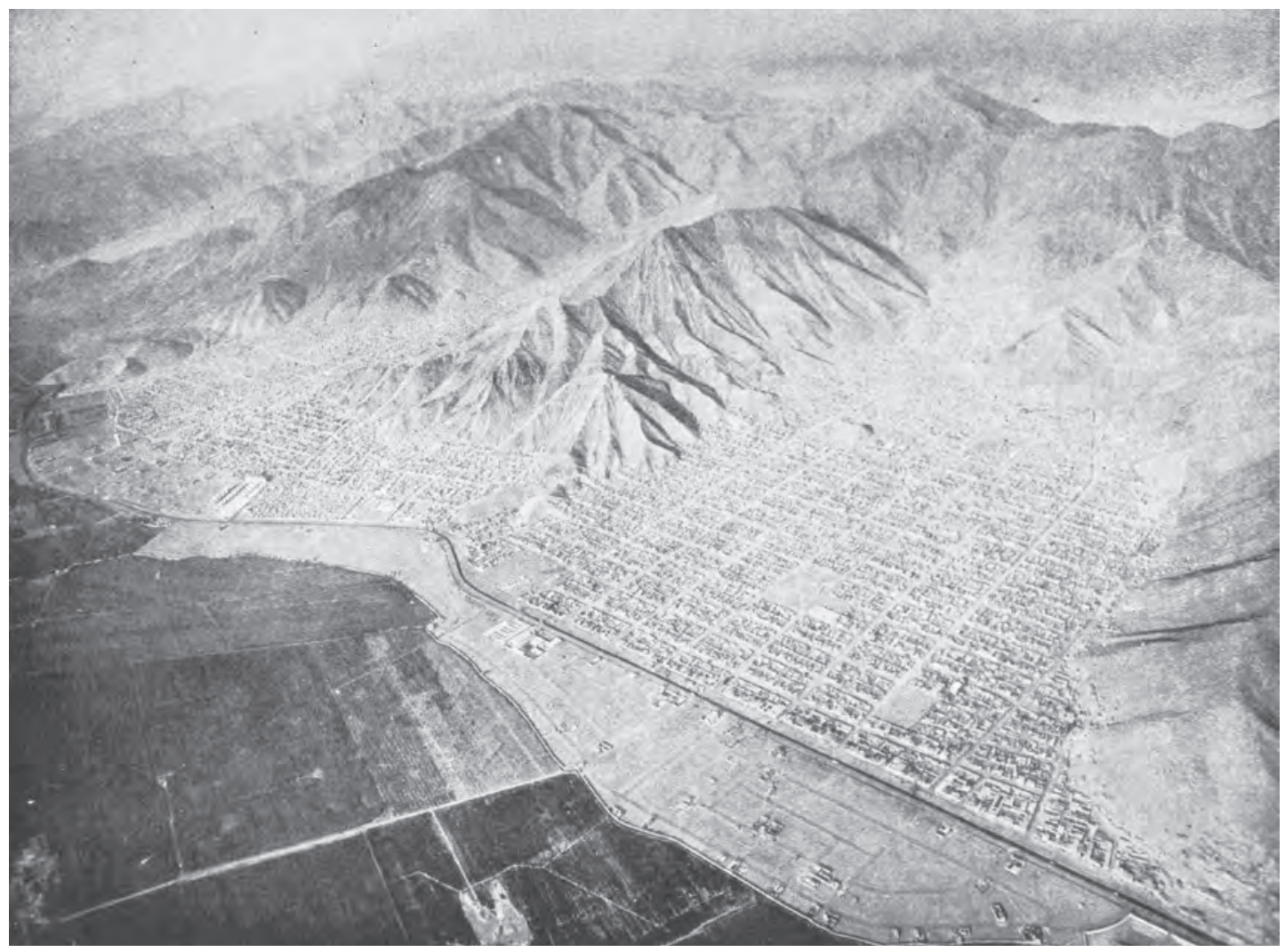

Figura 5. Pampa de Comas y Quebrada Boquerón durante el año de 1962, área para ese entonces poblada. Fotografía aérea oblicua realizada por el SAN (Smirnoff 1963:69).

Roselló describe los geoglifos en los siguientes términos: "La gran raya divergente en su parte superior, hacia el N-O [Noroeste], en lo que fue Pampa de Comas ... cuyos ambos lados en su término hacia el valle existieron cementerios del periodo ... [de los Centros Ceremoniales de Planta en 'U']' (Roselló 1997: 10).

El autor refiriéndose a los geoglifos de Pampa Canto Grande, precisa que el término raya debe entenderse como "una doble línea de ancho y largo variable, y el termino línea se usa solo cuando se trata de una sola" (Roselló et al. 1985: 44).

Una antigua nota periodística publicada en un diario de la capital, da cuenta del hallazgo de evidencias arqueológicas en la sección media de la pampa (Expreso 1963). Por el patrón observado en los complejos de geoglifos estudiados en la costa central, se trataría de una estructura asociada al geoglifo de Pampa de Comas.

Por otro lado, Roselló recupera material arqueológico del cementerio ubicado al lado sur asociado a la gran raya de Pampa de Comas atribuible al Horizonte Temprano, al igual que señala una marcada relación entre geoglifos, conglomerado de montículos y áreas de cementerios (Roselló 1997: 10, 42-43).

Asimismo, Bonavia (1966: 35) indica con el código PV46-50 un sitio arqueológico en el lado este de la avenida Túpac Amaru, a la altura del kilómetro 11 y a $100 \mathrm{~m}$ ascendiendo la Pampa de Comas, aproximadamente. El sitio fue caracterizado como un cementerio y atribuido al Horizonte Medio. Bonavia refiere esta información a Thomas C. Patterson, uno de los colaboradores de esta primera entrega de sitios arqueológicos, quien la fecha para el año de 1963. 
Tosso (2003), mediante el análisis de las fotografías aéreas de los años cuarenta, advierte de estructuras en la margen inferior norte de Pampa de Comas, nombrándolas de igual modo, las cuales describe como recintos de planta rectangular orientados de noroeste a suroeste. El mismo autor registra una confluencia de estructuras hacia el este de Pampa de Comas, en las inmediaciones de Cerro Almoguera denominándolas Complejo Interdistrital (Sitio de las Gradas, Sitio de Terrazas, Sitio de Alineamiento de Piedras, Sitio del Corral del Diablo, Sitio La Cama del Diablo, Sitio Los Corrales) los cuales describe como escalinatas, terrazas, muros, corrales y alineamiento de piedras a manera de recintos; elementos que recuerdan lo registrado en Pampa Canto Grande y sus quebradas subsidiarias (Abanto 2003, 2004, Abanto y García-Godos 2004).

\section{Conglomerados de montículos}

\section{Ubicación}

Roselló, al referirse al lado occidental de la cadena de cerros que divide la margen izquierda del valle bajo del río Chillón de la Pampa Canto Grande, advierte de conglomerados de montículos, señalando que aparecen asociados a los geoglifos y se ubican en el piso del valle, los cuales describe como "ligeros promontorios que sobrepasan lateralmente el ancho de la raya" (Roselló 1997:10), atribuyéndoles el carácter de ceremonial y aludiendo la filiación de estos conglomerados al Periodo Inicial y Horizonte Temprano.

El autor, mediante el análisis de las fotografías aéreas de la década de 1940, identifica el conglomerado de montículos frente a Pampa de Comas y asume una marcada relación entre ambos (Roselló 1997:10, 42-43). La referencia actual del mismo sería las urbanizaciones El Parral y Santa Luzmila ${ }^{16}$.

16 Villar (1982:171[1935]) se refiere al conglomerado de montículos frente a la pampa al detallar las ruinas de Comas. Tosso (2003) registra el asentamiento en el inventario de sitios arqueológicos con la denominación Complejo Santa Luzmila y lo subdivide a razón descriptiva.
La revisión de la Carta de Lima y alrededores: Hoja 5 f "Infantas" (escala 1:20,000; actualizada y reimpresa en 1962 del IGM) registra la presencia de montículos que se distribuyen desde las inmediaciones de Infantas con dirección hacia la Pampa de Comas, observándose mayor numero de los mismos entre la casa hacienda Comas y el límite de los campos con las tierras eriazas, ocupadas para ese entonces.

Sitios arqueológicos similares se han podido identificar en las fotografías aéreas utilizadas para el presente estudio, en los alrededores de Pampa la Encantada, al igual que en los terrenos contiguos al lado sur y suroeste de la Escuela de Aviación Civil Collique, frente a Quebrada Boquerón. Actualmente, se ubican en esta área las urbanizaciones de La Alborada y El Pinar.

\section{Descripción}

Silva (1996:253-257) registra estos asentamientos en el valle bajo y medio del río Chillón, denominándolos conglomerado de montículos y señala que estos sitios se componen de una serie de montículos, en número variable, construidos a manera de promontorios sólidos mediante el uso de cantos rodados, piedras canteadas y tierra, recubiertos por un muro de contención, carentes de estructuras interiores. La disposición de los mismos insinúan áreas similares a patios o plazas abiertas, indicando que las actividades que se realizaron tuvieron lugar alrededor o sobre los promontorios. No registra evidencia alguna de que estos hayan servido como estructuras sepulcrales y advierte la diferencia de algunos conglomerados, nombrándolos montículos efigie, dejando entrever su función residencial. Según el material constructivo y el material cerámico asociado, el autor atribuye ocupaciones en los conglomerados de montículos que van desde el formativo hasta los periodos tardíos.

Los sitios arqueológicos observados en el área de estudio presentan características similares comparables con los sitios registrados por Silva en La Pampilla, Oquendo, Chuquitanta, Caudivilla y Torreblanca en el valle bajo del río Chillón. 
Abanto (2004) describe un grupo de montículos similares en la parte baja de la quebrada Canto Grande, inexistente a la fecha, localizados en las fotografías aéreas de 1944, llamándolos Montículos Efigie de Zárate debido al lugar de referencia donde se ubicaron.

\section{Comentarios Finales}

Los sitios arqueológicos con presencia de geoglifos, materia de esta investigación, guardan semejanzas y contemporaneidad con los principales centros de Arte Rupestre en Cerro Panteón (valle medio del río Chillón), Pampa Canto Grande (valle bajo del río Rímac) y Quebrada Tinajas (valle bajo del río Lurín).

Los geoglifos, detallados en el presente estudio, fueron trazados sobre el fondo de las quebradas, en terrenos de poca pendiente, definidos mediante la limpieza del área y la alineación o acumulación de piedras, encajando dentro de la tipología de geoglifos ensayada por diversos investigadores para la costa central.

Accesos naturales de uso tradicional constituyen las quebradas en mención, que conectan el valle bajo del río Chillón con rutas que cruzaban Pampa Canto Grande, las cuales han quedado graficadas en croquis y cartas antiguas referentes al valle de Lima.

Entre ellos tenemos senderos que se internan por Pampa La Encantada, Quebrada Collique, pasando entre Cerro Tres Puntas, Cerro Mentira y el Cuello de Collique.

Igualmente, trochas habituales que se dirigen por Quebrada Boquerón, pasando por Portillo Malva, descendiendo por la quebrada y pampa de La Cruz.

Antiguas sendas que se distinguen en Pampa de Comas, remontando por Quebrada Piedra Grande, Cerro Almoguera y descendiendo por la quebrada homónima llegando a Pampa Canto Grande.

En lo que respecta al posible origen y significado de los geoglifos, se han ensayado diversas teorías, entre las que destaca la hipótesis astronómica, que plantea la interpretación de los geoglifos como instrumentos para observaciones astronómicas (Kosok 1965; Reiche 1993) y la hipótesis de explicar los geoglifos dentro de un contexto cultural, que establece una correlación entre geoglifos, cerros sagrados y sitios arqueológicos (Abanto 2003, 2004; Brignardello 2000, 2004, 2006; Engel 1983; Horkheimer 1947; Mejía 1942; Morrison 1978; Rodríguez 1997; Roselló 1997).

\section{Agradecimientos}

Al Lic. Julio H. Abanto Llaque, Presidente del Instituto Cultural Ruricancho, por la bibliografía brindada; al Fotógrafo Alejandro Balaguer, Presidente de la Asociación Andes y Mares, por la literatura relacionada con los trabajos del Lic. Carlos Brignardello Radulescu; al Arql. Francisco G. Vallejo Berríos por los datos referentes al plano de la Sociedad Geográfica de Lima de 1925; a la Bach. Cora A. Rivas Otaiza por la edición de las imágenes para el presente trabajo, por ultimo a los encargados de los Archivos de la Dirección General de Geografía del Instituto Geográfico Nacional y la Mapoteca del Centro de Investigaciones HistóricoMilitares del Perú por permitirme el acceso a la cartografía histórica.

\section{BiblogRAFÍA}

Abanto, J.

2003 "Nuevos Geoglifos en la Parte Alta de la Quebrada de Canto Grande. Valle del Rímac". Unay Runa 6:30-37. Lima.

2004 "Pictografía, Petroglifos y Geoglifos en la Quebrada Canto Grande, Valle del Rímac. Avances de su Estudio". Ponencia presentada en el I Simposio Nacional de Arte Rupestre. Cuzco.

Abanto, J. y M. García-Godos

2004 "Los Petroglifos Formativos de Cerro Cantería: Un adoratorio temprano entre la cuenca alta de Quebrada Canto Grande y Quebrada el Progreso, Lima”. 
Ponencia presentada en el Primer Encuentro Peruano de Arte Rupestre. Lima.

Brignardello, C.

2000 Simbología prehispánica del paisaje. Didi de Arteta. Lima.

2004 "El Paisaje Exento, The Missing Landscape". En Desiertos vivos: redescubriendo la costa del Perú. Living deserts : a rediscovery of the peruvian coast. pp. 105-223. Alva, W., A. Balaguer, J. Barrón, A. Benavides, C. Brignardello, R. Wiesse. Colección APU, AFP Integra. Lima

2006 "Geoglifos de Lurín, Geoglyphs of Lurin”. En Pachacámac: develando el misterio del valle de Lurín. Pachacámac: Unveiling the mistery of the Lurin valley, editado por A. Balaguer pp.37-47 Graph and Consult, Andes y Mares. Lima.

Bonavia, D.

1966 "Sitios Arqueológicos del Perú (Primera Parte)". Arqueológicas 9. Publicaciones del Instituto de Investigaciones Antropológicas. Museo Nacional de Antropología y Arqueología. Lima.

2000 "Las Pampas Misteriosas: Las Pampas de Nazca". En Culturas Prehispánicas, editado por Editora El Comercio, pp. 105 112. Lima.

Caretas

1995 ["Entre Líneas; cartas rectificatorias al artículo 'Líneas de Lima' (Zileri 1995)”] 1350, 16 de Febrero: Nos escriben ... y contestamos; 4, 6. Lima, Perú.

Centro de Investigación del Patrimonio Monumental. Facultad de Arquitectura, Urbanismo y Artes. Universidad Nacional de Ingeniería.

1988 Inventario del Patrimonio Monumental de Lima. Valles de Chillón, Rímac y Lurín. Tomo II, III (Época Prehispánica). Fundación Ford-Universidad Nacional de Ingeniería. Lima.

Cobo, B.

1956 [1653] Historia del Nuevo Mundo. Tomo II.

Biblioteca de Autores Españoles. Madrid.

Cogorno, G.

2006 "Tiempo de lomas: Calidades del medioambiente y administración de recursos en Lima, 1535-1601”. En Lima en el siglo
XVI, editado por L. Gutiérrez, pp. 19 102. Pontificia Universidad Católica del Perú-Instituto Riva Agüero. Lima

El Comercio

1977 "En Canto Grande, calendarios como en Nazca". 4 de Diciembre: El Dominical,12. 13. Lima, Perú.

Engel, F.

1983 "Líneas de Nazca, iTopografía, mitología o astronomía?". El Comercio. Diciembre 25:El Dominical, 6-7. Lima.

Expreso

1963 "Descubren palacio de piedra cubierto por arena del tiempo. 'Casa Azul' esta en Comas". 28 de Mayo:13. Lima, Perú.

Fondo Documentario de la Cultura Peruana. Universidad Nacional Federico Villarreal.

1998 "Tesauro del Patrimonio Cultural de Lima”. CD ROM Patrimonio Cultural del Perú. Vol. I. Lima.

Gutiérrez, B.

1992 "La Comunidad Campesina de Jicamarca; Migración y Control Territorial". Revista Peruana de Ciencias Sociales. 3(1):99-137. Lima.

Horkheimer, $\mathrm{H}$.

1947 "Las plazoletas, rayas y figuras prehispánicas en las pampas y crestas de la Hoya del Río Grande". Revista de la Universidad Nacional de Trujillo II(I):45-63. Trujillo.

1965 "Identificación y bibliografía de importantes sitios prehispánicos del Perú". Arqueológicas 8. Publicaciones del Instituto de Investigaciones Antropológicas. Museo Nacional de Antropología y Arqueología. Lima.

Hostnig, R.

2003 Arte Rupestre del Perú. Inventario Nacional. Universidad Nacional Mayor de San Marcos. Lima.

Huapaya, C.

1977-1978 "Vegetales como elemento antisísmico en estructuras prehispánicas". Arqueología PUCP 19-20:27-38. Pontificia Universidad Católica del Perú. Lima.

Kosok, P.

1965 Life, land and water in ancient Peru. Long Island University Press, New York. 
López, L.

1983 "Valor histórico de las Pampas de Canto Grande". Actualidad Militar 20(292):3435. Lima.

Ludeña, $\mathrm{H}$.

1975 Secuencia Cronológica y Cultural del Valle del Chillón. Tesis doctoral. Programa Académico de Arqueología, Universidad Nacional Mayor de San Marcos.

Mazzotti, L.

1973 "El Enigma de las Pampas de Nazca". Imagen 1(2):22, 48. Lima.

Mejía, T.

1942 "Acueductos y caminos antiguos de la Hoya del Río Grande de Nasca”. Actas y Trabajos Científicos del XXVII Congreso Internacional de Americanistas, tomo I, pp. 559-569. Lima.

1978 "Importancia prehistórica de la 'Huaca Florida' en el valle de Lima”. III Congreso Peruano El Hombre y la Cultura Andina. Actas y Trabajos, tomo II, editado por R. Matos, pp. 493-520. Lima

Milla, C.

1974 De Siná a Wallallo, Inventario y Catastro Arqueológico de los valles de Rimac y Santa Eulalia. Centro de Investigación y Restauración de Bienes Monumentales. Instituto Nacional de Cultura. Lima.

Morrison, T.

1978 Pathways to the Gods. The Mystery of the Nasca Lines. Andean Air Mail and Peruvian Times, Publishers. Lima.

Necochea, C.

1993 "Cerro de Canta guarda Riqueza Arqueológica”. El Comercio Febrero 21:D1. Lima.

1994 "Monumental Chillón”. El Comercio Abril 24:D1. Lima.

Pelikan, T.

1968 "The Cantogrande Valley". Peruvian Times 28(1427):2, 4. Andean Air Mail and Peruvian Times Publishers. Lima.

Petersen, G.

1980 Evolución y desaparición de las altas culturas Paracas - Cahuachi (Nasca). Universidad Nacional Federico Villarreal. Lima
Pinto, $M$.

2001 Informe Final. Proyecto de Evaluación Arqueológica de Reconocimiento sin Excavaciones V Zona Collique, Comas. Municipalidad Distrital de Comas.

La Prensa

1980 "Largos trazos que hay en Canto Grande serian de Mapa Astronómico Milenario". 23 de Enero:1. Lima, Perú.

Pulgar, J.

1962 Primera Exposición Nacional de Quilcas. Universidad Nacional Mayor de San Marcos. Lima.

1976 Quilcas, Arte Rupestre en el Perú. Universidad Nacional Mayor de San Marcos. Banco Continental del Perú. Lima.

Ravines, R.

1978 "Las misteriosas marcas terrestres de Canto Grande”. Oiga. 8:12

Reiche, M.

1993 Contribuciones a la Geometría y Astronomía en el Antiguo Perú. Asociación Maria Reiche para las Líneas de Nasca. Epígrafe Editores. Lima

Rodríguez, A.

1997 "Los campos de geoglifos en la costa central del Perú". Cuadernos de Investigación 2. Pontificia Universidad Católica del Perú-Instituto Riva Agüero. Lima.

1999 "Reconocimiento Aero-arqueológico del Complejo de Geoglifos de la Quebrada Torreblanca, Valle del Río Chillón. Costa Central del Perú". Boletín del Instituto Riva Agüero 26:297-314. Lima.

2002 "Geoglyfy u Limy: Tanec kolem mytického hada [Geoglifos de los alrededores de Lima: el baile de la serpiente mítica]". Vesmir 81: 98-99. Czech.

Rosselló, L.

1978 "Sistemas astronómicos de campos de rayas". III Congreso Peruano El Hombre y la Cultura Andina. Actas y Trabajos, tomo II, editado por R. Matos, pp. 521 534. Lima

1997 Cantogrande y su relación con los Centros Ceremoniales de Planta en U". Arqueología de la Costa Central. Mundo Grafico. Lima. 
Rosselló, L., C. Huapaya y L. Mazzotti

1985 "Rayas y Figuras en la Pampa de Canto Grande". Boletín de Lima.7(39):41-58. Lima.

Rostworowski, M.

1972 "El sitio arqueológico de Concon, en el valle del Chillón: derrotero etnohistórico". Revista del Museo Nacional 38:315 326

Silva, J.

1996 Prehistoric Settlement patters in the Chillon Valley, Peru. Ann Arbor, University of Michigan. Department of Anthropology. Doctor Anthropology.

2002 "Reseña de Cantogrande y su relación con los Centros Ceremoniales de Planta en 'U'. Arqueología de la Costa Central". Bulletin de l'Institut français d'études andines 31(2):409-411.

Smirnoff, V.

1963 "25 Años de vivienda en el Perú”. El Arquitecto Peruano, 306-307-308:44-81. Lima
Tácunan, S.

2000 Comas y su historia: un modelo de historia distrital. Biblioteca Nacional del Perú. Lima

Thommen, P.

1946 "Extraños dibujos en la Pampa de Nazca”. El Arquitecto Peruano Año XI, No113, Diciembre. Lima

Tosso, W.

2003 Proyecto Comas-Inventario y Registro Arqueológico del Distrito de Comas. Municipalidad Distrital de Comas. Lima.

Valls, E.

1980 "Enigma de Nazca se repite en Lima". Ultima Hora Febrero 23:8. Lima.

Villar, P.

1982 [1935] Arqueología del Departamento de Lima; Las Culturas Prehispánicas del Departamento de Lima. Atusparia. Lima.

Zileri, D.

1995 "Líneas de Lima”. Caretas N 1348, Febrero 2. pp. 90-91 